\title{
ESTIMATES OF BOLOMETRIC CORRECTIONS FOR WR STARS AND PROGENITORS OF WN BINARIES.
}

\author{
C. DE LOORE and J.P. DE GREVE \\ Astrophysical Institute, V.U.B. \\ Pleinlaan 2, B - 1050 Brussels, Belgium.
}

\begin{abstract}
.
Using theoretical models of WR binary evolution, and adopting bolometric corrections for the O-type companions, the B.C. of a sample of WN stars are derived using the luminosity difference and the mass ratio. From the present characteristics of observed WN binaries the initial parameters are estimated assuming conservative case $B$ of mass transfer and stellar wind as mass modifying processes. The influence of nonconservative interaction is discussed.
\end{abstract}

\section{Evolutionary models and observed binaries.}

For a number of Wolf-Rayet binaries the masses, period and mass ratio are reasonably well known. We adopted the values given by van der Hucht et al. (1988), other resembling values can be found in Schulte-Ladbeck (1989) and Smith and Maeder (1989). We further calculated the mass ratio evolution of binary systems after a conservative case B of mass transfer, for initial primary mass values between 15 Mo and $80 \mathrm{Mo}$, and mass ratios $\mathrm{qi}=0.6$ and 0.9 . The luminosity evolution of the WR star was calculated using models of helium stars by Langer (1989a). For the calculation of the mass we adopted his mass dependent mass loss formalism (Langer, 1989b). The mass of the O-type companion was kept constant in view of the short timescale involved, and the much lower mass loss rate.

Table 1. Characteristics of WN $+\mathrm{O}$ binaries (masses are in $\mathrm{M}_{\mathbf{O}}$ ).

\begin{tabular}{|c|c|c|c|c|c|c|c|}
\hline $\mathrm{HD}$ & WR & Type & $P(d)$ & $\mathrm{M}(\mathrm{WR})$ & $\mathrm{M}(0)$ & R) -1 & VR) \\
\hline 90657 & 21 & WN4 + 046 & 8.26 & 12 & 24 & 3.5 & 5.7 \\
\hline 94546 & 31 & WN4 + 07 & 4.83 & 9 & 17 & 3.5 & 5.7 \\
\hline 190918 & 133 & WN4.5 +09.5 & 112.8 & 15 & 15 & 5.1 & 3.5 \\
\hline CX Cep & 151 & WN5 + O8V & 2.13 & 6 & 14 & 4.8 & 3.6 \\
\hline 193576 & 139 & WN5 + O6 & 4.21 & 10 & 26 & 5.1 & 3.6 \\
\hline E311884 & 47 & WN6 + O5 & 6.34 & 43 & 51 & 5.5 & 5.3 \\
\hline 186943 & 127 & WN4 + $09.5 \mathrm{~V}$ & 9.55 & 16 & 35 & 3.7 & 5.7 \\
\hline
\end{tabular}

453

K. A. van der Hucht and B. Hidayat (eds.),

Wolf-Rayet Stars and Interrelations with Other Massive Stars in Galaxies, 453-457.

(C) 1991 IAU. Printed in the Netherlands. 
Its luminosity was calculated from the following average main sequence mass-luminosity relation

$$
\log \mathrm{L} / \mathrm{L}_{\mathrm{O}}=1.522+2.468 \log \mathrm{M} / \mathrm{M}_{\mathrm{O}}
$$

derived from our computations for single stars (Figueiredo et al., 1990, preprint).

The results for the mass and the luminosity ratio between the components are shown in Figure 1.

Observed WN + $\mathrm{O}$ binaries with rather well known characteristics, are given in table 1 . The data are taken from van der Hucht et al. (1988), except for HD 90657 and 94546, where we used the results of Schulte-Ladbeck (1989), but completed with the general BC-values for WN4 stars quoted by van der Hucht et al.

In the following $q_{i}$ refers to the initial mass ratio and is defined as $q_{i}=M_{2 i} / M_{1 i}$, qf refers to the inverse mass ratio after mass transfer, defined as $\mathrm{q}_{\mathrm{f}}=\mathrm{M}_{1} / \mathrm{M}_{2} \approx \mathrm{M}(\mathrm{WR}) / \mathrm{M}(\mathrm{O})$.

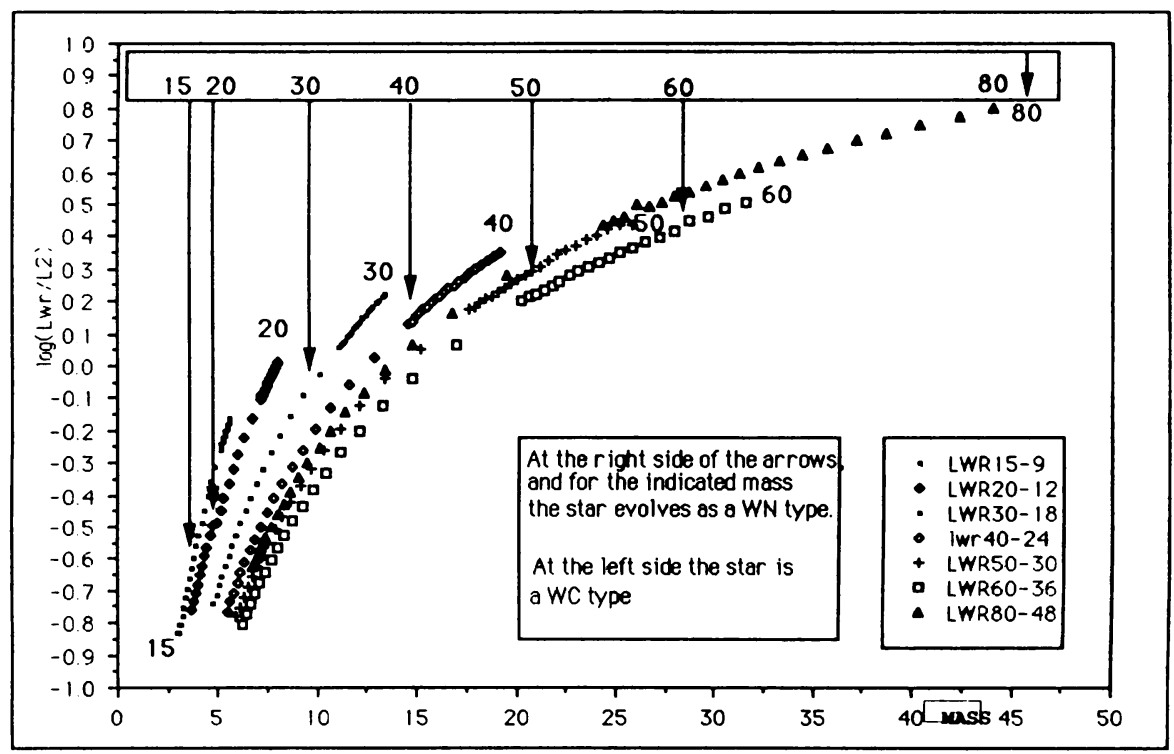

Figure 1. Luminosity ratio (in $\log$ ) as a function of the mass, for massive binary systems after a case $B$ of mass transfer. Initial masses are in the range 15 to $80 \mathrm{M}_{\mathrm{O}}$. Vertical arrows indicate the boundary between WN and WC phase.

\section{Bolometric corrections for the WN components.}

We assume that the mass ratio and the bolometric corrections of the $\mathrm{O}$-stars are accurately known (with respect to the $B C$ of the WR star).

The $B C$ of the WR star can be expressed as a function of $B C(O), M_{V}(W R)$ and the ratio of the luminosities of the two components, using the well known bolometric magnitude-luminosity conversion. The resulting relation is

$$
\mathrm{BC}(\mathrm{WR})=\mathrm{M}_{\mathrm{b}}(\mathrm{O})-\mathrm{M}_{\mathrm{v}}(\mathrm{WR})-2.5 \log (\mathrm{L}(\mathrm{WR}) / \mathrm{L}(\mathrm{O}))
$$


We plotted the log of the ratio of the luminosities as a function of the mass ratios (for two initial values of $\mathrm{q}_{\mathrm{i}}$ ). Figure 2 shows the result for $\mathrm{q}_{\mathrm{i}}=0.6$. The theoretical models occupy a narrow band in the diagram, with $\Delta \log L(W R) / L(O)$ ranging from $0.05(q=0.15)$ to $0.14(q=0.45)$. Using the values of table 1 , the observed WN binaries were plotted in the same diagram. We found that they are located well outside the theoretical band. The largest vertical difference 0.8 , was found for WR 127, the smallest, 0.12 , for WR 139. Next, we assumed that the differences were due to errors in the estimation of the BC of the WR stars (and not in the other parameters involved). We then derived a new estimate of $B C(W R)$ requiring the observed systems to fall on the mean of the theoretical $\log L(W R) / L(O)$ - mass ratio relation. The resulting BC values and related quantities, are given in table 2.

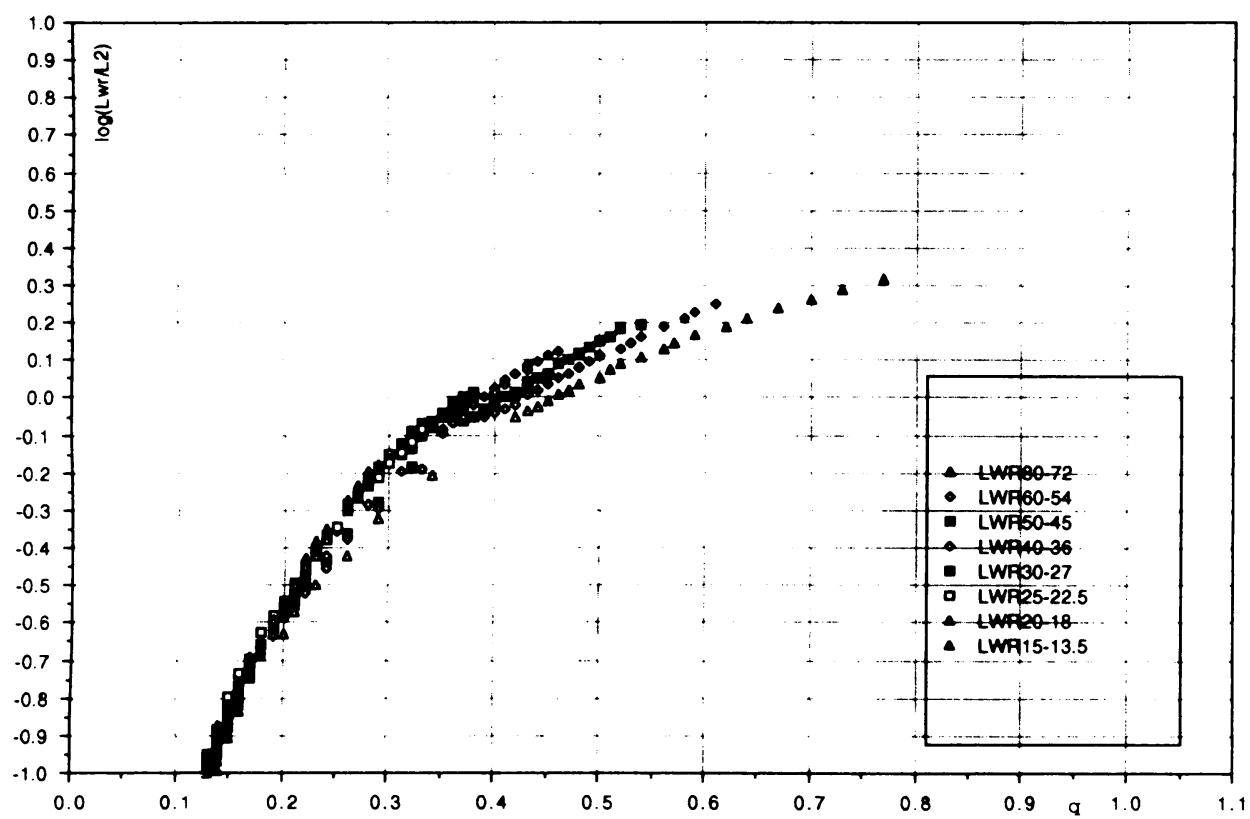

Figure 2. Luminosity ratio (in log) as a function of the mass, for massive binary systems after a case $B$ of mass transfer, for $\mathrm{q}_{\mathrm{i}}=0.6$, and initial masses $\mathrm{M}_{1 \mathrm{i}}=15$ to $80 \mathrm{M}_{0}$.

Although this method has the advantage of using a narrow relation with mostly well known quantities, it has the disadvantage of being dependent on the initial mass ratio.

However, except for WR47 and WR127, the differences between the two BC values for $q_{i}=0.6$ and $q_{i}=$ 0.9 are less than $8 \%$ of the value for $q_{i}=0.6$ (see the last column in table 2 ).

The theoretical luminosity ratio and the mass ratio also depend on the assumption on the mode of mass transfer. Nonconservative mass transfer results in a smaller value for the mass of the $O$ star, hence a larger mass ratio. It also results in a lower luminosity for this component, hence a larger value of log $\mathrm{L}(\mathrm{WR}) / \mathrm{L}(\mathrm{O})$. We calculated the shift of the theoretical luminosity ratio-mass ratio relation, assuming half of the transferred mass is leaving the system. Due to the fact that the points move upward and to the right of the diagram, the overall difference between the new relation and the relation for conservative 
mass transfer is extremely small (and hence the bolometric corrections). We therefore conclude that, unless the mass transfer is highly non-conservative, or the initial mass transfer very small, the bolometric corrections for WN stars in binaries are fairly well represented by the values derived from models with $q_{i}=0.6$ adopting conservative mass transfer.

The average value of $\mathrm{BC}(\mathrm{WR})$ from our results is -4.6 , which corresponds well with the value quoted by Smith and Maeder (1989). Also the results for the individual systems correspond quite well. The differences with the results of van der Hucht et al. arise from the use of different models and their use of the mass of the WR star, a quantity much less well known than the mass ratio.

Table 2. Bolometric corrections for WN stars, derived from the theoretical $\log L(W R) / L(O)$ - mass ratio relation for $\mathrm{q}_{\mathrm{i}}=\mathbf{0 . 6}$ The corresponding bolometric magnitudes and luminosities are also given. The last column shows the values derived using theoretical models with $\mathrm{q}_{i}=0.9$.

\begin{tabular}{rccccc}
\hline WR & $-\mathrm{BC}$ & $\mathrm{M}_{\mathrm{V}}(\mathrm{WR})$ & $-\mathrm{M}_{\mathrm{b}}(\mathrm{WR})$ & $\log \mathrm{L}(\mathrm{WR}) / \mathrm{L}_{0}$ & $\mathrm{BC}(0.9)$ \\
\hline 21 & 5.8 & 3.5 & 9.3 & 5.60 & 5.55 \\
31 & 5.0 & 3.5 & 8.5 & 5.28 & 5.25 \\
133 & 4.5 & 5.1 & 9.6 & 5.72 & 4.15 \\
139 & 3.85 & 5.1 & 8.95 & 5.42 & 3.6 \\
151 & 3.55 & 4.8 & 8.35 & 5.22 & 3.3 \\
47 & 5.30 & 5.5 & 10.8 & 6.20 & 4.44 \\
127 & 4.35 & 3.7 & 8.05 & 5.09 & 3.53 \\
\hline
\end{tabular}

\section{Progenitors of WN binaries}

Using the foregoing results for the WN stars, we now determine the initial mass and mass ratios of these systems. To obtain these, we compared the mass and luminosity of the WN star with the theoretical models (for $\mathrm{q}_{\mathrm{i}}=\mathbf{0 . 6}$ and 0.9 ) and looked for the best agreement. The same was done for the mass and mass ratio. The initial parameters leading to the best correspondence (after a conservative case $B$ of mass transfer) between theory and observations in the (M, q, L)-space, were considered as progenitors. The results are given in Table 3.

Under the assumption of conservative case $B$ mass transfer (a combination of 3 assumptions : mass transfer, case $B$, conservative !) the WN stars have progenitor masses around $30 \mathrm{M}_{\mathrm{O}}$ (except WR 47) and an initial mass ratio larger or equal to 0.7 (except WR 127 and WR 47). Adopting a nonconservative mode of mass transfer results in larger mass ratios $M(W R) / M(O)$ after mass transfer. If we assume that half of the transferred mass is leaving the system, the final mass ratio increases by 0.17 (for $M_{1 i}=15$ $\left.\mathrm{M}_{0}\right)$ to $0.2\left(\mathrm{M}_{1 \mathrm{i}}=40 \mathrm{M}_{0}\right)$. This in turn results in larger initial mass ratios for the observed WN binaries. Hence, nonconservative mass transfer brings the initial mass ratio close to 1 for all systems (again with exception of WR47).

\section{Acknowledgement.}

This work has been supported by the National Fund of Scientific Research (NFWO - Belgium) under grant No. S 2/5 - LV. E96. 
Table 3. Progenitors systems for $\mathrm{WN}$ binaries, from the comparison of present mass, mass ratio and luminosity with models after a conservative case B of mass transfer.

\begin{tabular}{lccc}
\hline WR & M1i/Mo & M2i/Mo & Actual WN state \\
\hline 21 & 35 & 28 & mid \\
31 & 28 & 22 & mid \\
151 & 30 & 21 & begin \\
127 & 30 & 15 & begin \\
139 & 30 & 27 & end \\
47 & 60 & 24 & end \\
133 & 30 & 21 & end \\
\hline
\end{tabular}

\section{References}

Figueiredo, J., De Greve, J.P., de Loore, C. : 1990, submitted to A \& A

Langer, N. : 1989a, Astron. Astrophys. 210, 93.

Langer, N. : 1989b, Astron. Astrophys. 220, 135.

Schulte-Ladbeck, R.E. : 1989, Astron. J. 97, 1471.

Smith, L., Maeder, A. : 1989, Astron. Astrophys. 211, 71.

van der Hucht, K.A., Hidayat, B., Admiranto, A.G., Supelli, K.R., Doom, C. : 1988, Astron.

Astrophys. 199, 217.

\section{DISCUSSION}

Pakull: I would like to remind you that for some of these stars which have $\mathrm{HeIII}$ regions around, we can really determine the luminosity and also the bolometric correction in this case, and for these two stars (whatever you call them, WN1 or WN2) which have HeII4686 nebulae around, we derive the bolometric correction, and we find for one of these stars -5.6 and for the other one -7 . That is a direct measurement, there is no assumption in it, except that it radiates as a black body, but I was told from the people who do the models that a black body is not a bad approximation for these kinds of stars.

Schmutz: It appears that your B.C.'s are consistent with what we have determined from the spectroscopic analyses. 


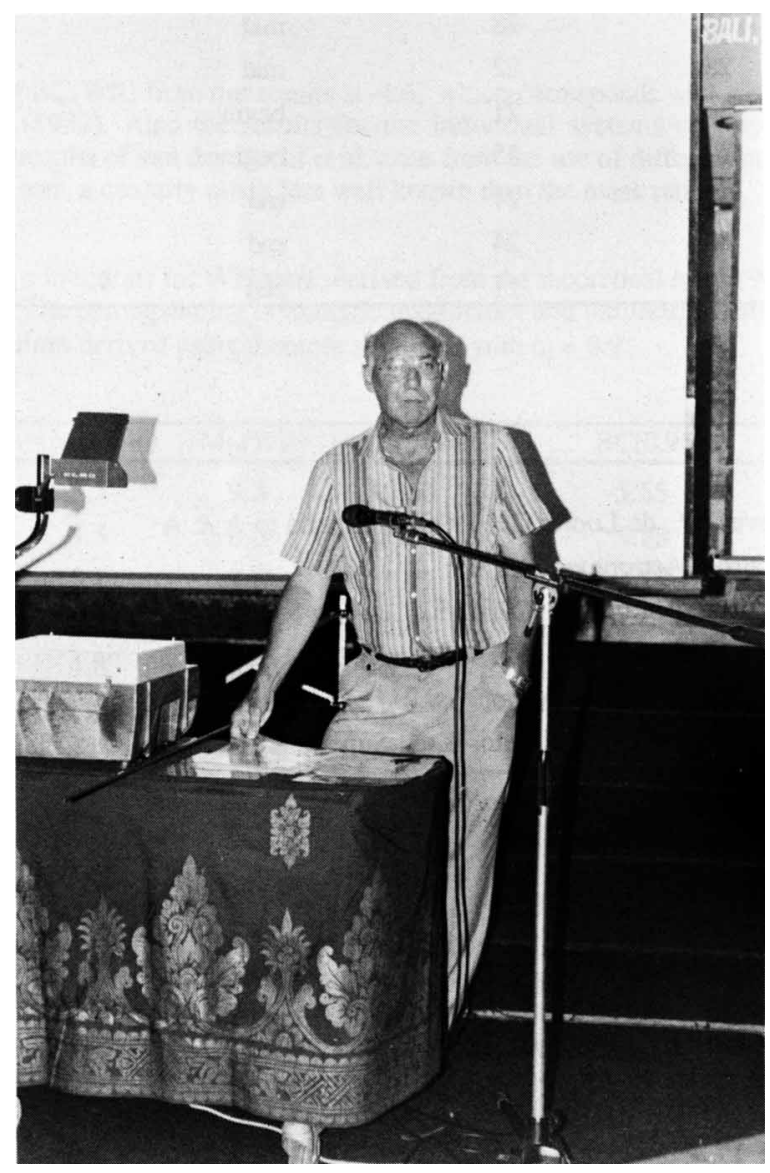

Bert de Loore 